\title{
DESIGN AND FABRICATION OF INP FREE-STANDING OPTICAL WAVEGUIDES FOR MEMS
}

\author{
Wing $\mathrm{H} \mathrm{Ng}^{1}$, Nina Podoliak ${ }^{2}$, William Stewart ${ }^{2}$, Peter Horak ${ }^{2}$, \\ Huiyun Liu ${ }^{1}$ and Anthony J Kenyon ${ }^{1}$ \\ ${ }^{1}$ Department of Electronic \& Electrical Engineering, University College London, UK \\ ${ }^{2}$ Optoelectronics Research Centre, University of Southampton, UK \\ E-mail: w.ng@ucl.ac.uk
}

\begin{abstract}
We present the design and fabrication of an optical MEMS device on InP platform. The device is based on a suspended parallel waveguide configuration with side pillars supports. Electrodes are integrated on the device layer to provide MEMS actuation functionality to the waveguides. The device is designed to be used as an optical buffer for telecommunication applications.
\end{abstract}

\section{INTRODUCTION}

It has been reported recently that a continuously tunable optical buffer can be realized using two actuated waveguides [1]; theoretical calculations were based on using silicon as the active material. In this paper, we report the design and fabrication of such a device on an InP platform. III-V materials such as InP and GaAs have several major advantages over silicon based materials. In terms of optical properties, optical gain can be achieved in III-V materials, due to the direct nature of their bandgap. From a technological point of view, lasers and other optical components for optical communications are well established in the InP platform [2], and hence our device can be monolithically integrated into the same circuit.

\section{WAVEGUIDE DESIGN}

The design for an optical buffer [1] requires two free-standing optical waveguides with an optimal size of $200 \mathrm{~nm} \times 300 \mathrm{~nm}$. By MEMS actuation the separation between the waveguides can be changed continuously between 50nm and 500nm leading to up to $100 \%$ change of the pulse propagation delay time.

In order to achieve substantial delay times, a long device is required, e.g., $1 \mathrm{~mm}$ length for a $10 \mathrm{ps}$ delay. Minimizing propagation losses is therefore of paramount importance and various loss mechanisms need to be considered. First, surface roughness as determined by the fabrication procedure leads to light scattering and losses of typically $1 \mathrm{~dB} / \mathrm{cm}$. To minimize these losses we consider a TE (y-polarization in Figure 1) rather than a TM (x-polarization) mode for operation, as this mode has weaker electric fields at the side walls.
For the envisaged buffer functionality, a significant fraction of light must be propagating in the air around the waveguides, which may lead to leakage of power into any nearby material. Numerical simulations suggest that to keep these losses below $1 \mathrm{~dB} / \mathrm{cm}$ the waveguides should be suspended $\sim 3 \mu \mathrm{m}$ above the substrate and $\sim 4 \mu \mathrm{m}$ away from any side walls.

The pillar supports required for the suspension of the waveguides will also introduce additional scattering and leakage of light. The waveguides are therefore designed with S-shaped tapers at the pillar connections as shown in Figure 1, confining the light more strongly inside the tapers and thus reducing losses at the pillar connection. A loss of $\sim 0.2 \mathrm{~dB}$ per pillar is calculated for tapers of $600 \mathrm{~nm}$ thickness and $7-8 \mu \mathrm{m}$ length, while a loss $>1 \mathrm{~dB}$ is expected without tapers. S-shaped sections are designed on both waveguides at each pillar attachment point to ensure symmetrical optical modes propagating.

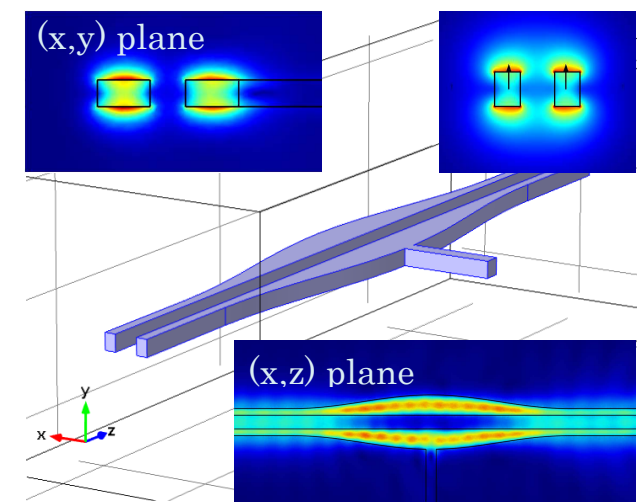

Figure 1. Geometry of suspended waveguides with S-shaped tapers at the pillar connection. Insets: electric field profile of TE mode in the waveguides (right) and in the S-shaped taper (left).

\section{FABRICATION}

The sample was grown on an n-type InP substrate by Molecular Beam Epitaxy (MBE). The device layer consisted of a $300 \mathrm{~nm}$ layer of InP. A $3 \mu \mathrm{m}$ layer of $\mathrm{In}_{0.57} \mathrm{Ga}_{0.43}$ As was sandwiched between the device layer and the substrate. This served as a sacrificial layer, etching of which allowed the waveguide structures written into the InP layer to be released. The sample structure is shown in Figure 2. 


\begin{tabular}{|c|}
$0.3 \mu \mathrm{m} \operatorname{InP}$ (Device Layer) \\
\hline $3 \mu \mathrm{m} \operatorname{InGaAs}$ (Sacrificial Layer) \\
\hline n-type InP Substrate \\
\hline
\end{tabular}

Figure 2. Layer structure of the InP MEMS waveguides.

The device was fabricated on a $1 \mathrm{~cm}$ by $1 \mathrm{~cm}$ InP chip. Following deposition of the $\operatorname{In}_{0.57} \mathrm{Ga}_{0.43} \mathrm{As}$ sacrificial layer, the waveguides, supporting side pillars and electrode pads were all fabricated on the same InP device layer.

The patterning of the gold electrode pads was done by using electron beam lithography (Raith150-TWO). The gold electrodes were deposited by thermal evaporation and lift-off in acetone.

The patterning of the parallel waveguides was carried out using hydrogen silsesquioxane (HSQ) electron beam resist as a negative mask. The dimensions of the written waveguides were $250 \mathrm{~nm}$ in width and $200 \mu \mathrm{m}$ in length. The spacing between the waveguides was approximately $300 \mathrm{~nm}$. Pillars were patterned in the same step as the waveguides. The spacing between pillar supports was $40 \mu \mathrm{m}$, and each pillar was composed of two parts: an S-shaped area that attached to the waveguide and a rectangular section that linked the S-shaped area to the large contact pad. The length of the S-shaped area was approximately $5 \mu \mathrm{m}$ and the width was $500 \mathrm{~nm}$. The length of the rectangular section was $3 \mu \mathrm{m}$. Pillar positions on the waveguides were staggered to allow the waveguides to be pulled apart by the applied field. A section of the pattern is shown in Figure 3.

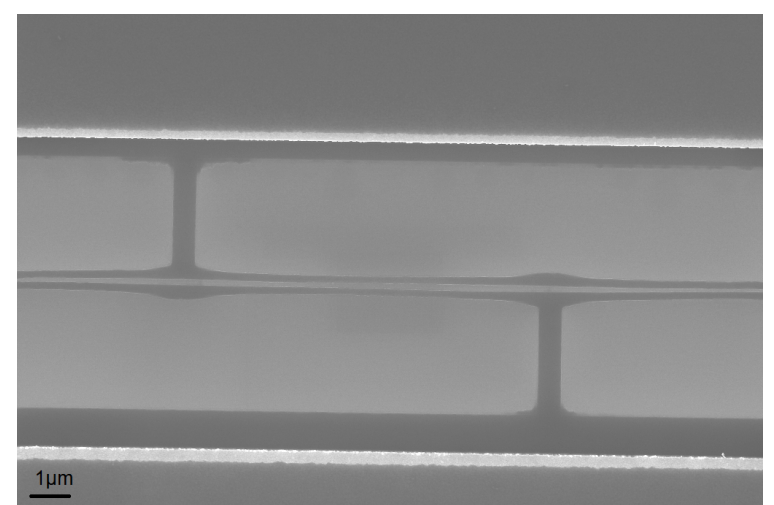

Figure 3. Top view of the waveguide and pillar pattern.

After the waveguides and pillars were patterned, the device layer was etched by reactive ion etching (RIE) with a cyclic methane-hydrogen/oxygen plasma.
The resulting etched structure has a sidewall profile better than 80 degrees. The waveguides were released using $\mathrm{HF}: \mathrm{H}_{2} \mathrm{O}_{2}: \mathrm{H}_{2} \mathrm{O}(1: 1: 8)$ solution. This step etches away the $\mathrm{In}_{0.57} \mathrm{Ga}_{0.43}$ As layer with the mask cleanly, without leaving any remnant on the waveguide surfaces.

\section{RESULTS}

Figure 4 shows an SEM image of the released waveguide structure. The dimensions of each waveguide were approximately $300 \mathrm{~nm}$ (height) $\mathrm{x}$ 200nm (width) x 200 $\mu$ m (length). The gap between the waveguides was approximately 400nm. The free standing waveguides did not collapse after release.

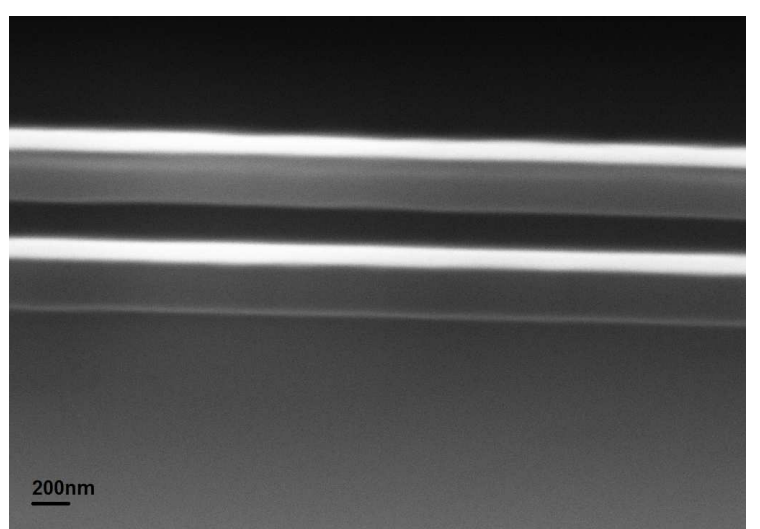

Figure 4. Free-standing waveguides after HF etch.

\section{CONCLUSION}

We have demonstrated the design and fabrication of suspended waveguides on an InP platform with MEMS functionality. Current and future work will focus on optimizing the fabrication steps to match closely the designed dimensions, and demonstrate full functionality as a tunable optical buffer.

This work was supported by the EPSRC "Continuously tunable optical buffer" project (EP/J012823/1 and EP/J012874/1).

\section{REFERENCES}

[1] P. Horak, W. Stewart, W. H. Loh "Continuously tunable optical buffer with a dual silicon waveguide design" Optics Express, vol. 19, no. 13, pp. 12456-12461, 2011.

[2] F. Lelarge et al., "Recent advances on InAs/InP quantum dash based semiconductor lasers and optical amplifiers operating at $1.55 \mu \mathrm{m}$ " IEEE Journal of Selected Topics in Quantum Electronics, vol. 13, pp. 111-124, 2007. 